\title{
Two-Stage Dynamic Sensor Deployment Strategy Based on Virtual Force and Genetic Algorithm in Wireless Sensor Networks
}

\author{
${ }^{\mathrm{a}}$ Jianguo Shi, ${ }^{\mathrm{b}}$ Changjie Zhou \\ ${ }^{a}$ Zhongshan Institute University of Electronic Science and Technology of China Zhongshan, China \\ ${ }^{b}$ School of Science Hebei University of Science and Technology Shijiazhuang, China
}

\begin{abstract}
Dynamic sensor deployment is one of the key topics in the research of WSNs. The performance of virtual force algorithm may be deteriorated because the stationary sensor nodes will confine the global optimal searching ability. Genetic algorithm is an efficient optimization tool for multi-dimensional optimization problems in a continuous space with some disadvantages such as slow convergence and prematurity. This paper proposes a two-stage dynamic sensor deployment strategy in WSNs based on virtual force and genetic algorithm. That is, the algorithm firstly deploys the dynamic sensors in continuous larger area in WSNs in an approximate optimal way to produce high quality initial population by virtual force. Then GA is employed to achieve global optimization coverage of WSNs based on the result of the first stage. Simulation results demonstrate that the algorithm presented in this paper is effective and efficient.
\end{abstract}

Index Terms: wireless sensor networks; coverage; mobile node; virtual force; genetic algorithm

(C) 2012 Published by MECS Publisher. Selection and/or peer review under responsibility of the International Conference on E-Business System and Education Technology

\section{Introduction}

Wireless sensor networks are widely used in target tracking, military surveillance, environment monitoring and other fields. Optimal deployment according to monitoring environment can improve the coverage rate of wireless sensor networks (WSNs) and the target detection rate, and it can also reduce the consumption of network energy. Optimal deployment has become one of the key issues in performance optimization for the research of WSNs. Many scholars have studied this problem, for example, Wang proposes VOR approach [1], Howard proposes artificial potential approach [2], Zou proposes virtual force approach [3] and Tian Yiming proposes multiobjective nonlinear programming model method [4] etc. In these studies, the virtual force algorithm received wide attention. However, the virtual force of fixed sensor nodes on mobile sensor nodes may limit the power of the layout of global optimization in WSNs which is composed by fixed sensor nodes and mobile sensor nodes. To solve this problem, Xue Wang [5] proposes dynamic sensor deployment strategy based on virtual force-directed particle swarm optimization and parallel particle swarm optimization based mobile sensor node deployment in WSNs, and Jie Jia [6] proposes optimal coverage scheme based on genetic algorithm 


\section{Wireless Sensor Networks}

in WSNs. Although the particle swarm optimization and genetic algorithm can effectively optimize the layout of a wireless sensor network, it also has problems in the computation process, such as slow convergence and premature convergence.

To solve the above problem, this paper proposes a two-stage dynamic sensor deployment strategy in WSNs based on virtual force and genetic algorithm (VFGA). That is, the algorithm firstly deploys the dynamic sensors in continuous larger area in WSNs in an approximate optimal way to produce high quality initial population by virtual force. Then GA is employed to achieve global optimization coverage of WSNs based on the result of the first stage. This strategy not only avoids the constrained optimization of mobile sensor nodes that is resulted by virtual force algorithm, but also makes population genetic algorithm evoluting to the direction of expanding the coverage and target detection rates. This strategy improves the coverage and convergence speed. Simulation results demonstrate that the algorithm presented in this paper is effective and efficient.

\section{Sensor deployment strategy based on VFGA in wireless sensor networks}

Genetic algorithm is an intelligent optimization method, and it has the advantage at speed and robustness etc. But for some complicated issues, its convergence is slow, and it is easy to premature convergence that can be trapped in local optima. Based on this thinking, this paper designs several optimization strategies according to the characteristics of virtual force algorithm and genetic algorithms and carries out a comparative study[7]:

\subsection{Real-coded genetic algorithm (RCGA)}

The following is the steps of coverage optimization strategy in wireless sensor network based on RCGA.

1) Initialized: Set the parameters of population size, evolution generation, crossover probability, mutation probability etc.

2) Cross: Cross-process uses heuristic crossover operator and uses crossover probability to randomly select two parent individuals. It can be denoted as:

$x^{1}=\left(x_{1}^{1}, x_{2}^{1}, \cdots, x_{n}^{1}\right)^{T}, x^{2}=\left(x_{1}^{2}, x_{2}^{2}, \cdots, x_{n}^{2}\right)^{T}$

Offspring Individuals can be produced by the following formula:

$y= \begin{cases}x^{1}+\Gamma\left(x^{1}-x^{2}\right) & \text { fit }\left(x^{1}\right) \leq f i t\left(x^{2}\right) \\ x^{2}+\Gamma\left(x^{2}-x^{1}\right) & \text { fit }\left(x^{1}\right)>\operatorname{fit}\left(x^{2}\right)\end{cases}$

In which, $\Gamma=\operatorname{diag}\left(\gamma_{1}, \cdots, \gamma_{n}\right), \gamma_{i} \in(-1,1)$ and $\gamma_{i} \neq 0, i=1, \cdots, n$.

3) Variation: Transformation process uses uniform mutation operator, and each variable of each individual has equal variance opportunity.

4) Choice: Uses roulette wheel selection operator.

5) Elitist strategy and termination conditions.

\subsection{Two-stage strategy based on virtual force and genetic algorithm (VFGA}

Ergodicity and diversity of the initial population has a key influence to the performance of genetic algorithm. VFGA algorithm is divided into two stages. In the first phase we use VF algorithm to Local approximation optimal cover in a larger area[8]. In the second stage we use the approximate optimal coverage solution of local 
area that is received from the first stage to initialize the population of RCGA algorithm, and then implement coverage optimization of the entire region[9,10].

\subsection{Genetic algorithm based on virtual force blend crossover operator (VFCGA)}

In order to speed up the convergence rate of genetic algorithm in the optimization process and guide the evolution of genetic algorithm to the global optimum, VFCGA algorithm introduces the influence of virtual force during the individual cross of RCGA algorithm.

$y= \begin{cases}x^{1}+\Gamma\left(x^{1}-x^{2}\right)+v x_{\text {move }}^{1} & \text { fit }\left(x^{1}\right) \leq f i t\left(x^{2}\right) \\ x^{2}+\Gamma\left(x^{2}-x^{1}\right)+v x_{\text {move }}^{2} & \text { fit }\left(x^{1}\right)>f i t\left(x^{2}\right)\end{cases}$

$v$ is influence coefficient of Virtual force. $x_{\text {move }}^{1}$ and $x_{\text {move }}^{2}$ are the traveled distance of cross generations infulenced by virtual force. They can computed By (3) and (4), and the meaning of other parameters is the same as (1).

$X_{\text {move }}= \begin{cases}0 & \left|F_{x y}\right| \leq F_{t h} \\ \frac{F_{x}}{F_{x y}} \times \text { MaxStep } \times e^{-\frac{1}{F_{x y}}} & \left|F_{x y}\right|>F_{t h}\end{cases}$
$y_{\text {move }}= \begin{cases}0 & \left|F_{x y}\right| \leq F_{t h} \\ \frac{F_{y}}{F_{x y}} \times \text { MaxStep } \times e^{-\frac{1}{F_{x y}}} & \left|F_{x y}\right|>F_{t h}\end{cases}$

\subsection{Genetic algorithm based on virtual force blend mutation operator (VFMGA)}

Niche genetic algorithm is an effective method to solve the problem of multimodal function global optimization. It can overcome premature convergence that is caused by the decrease of population diversity in the evolution process of genetic algorithm. Meanwhile, the $(\mu+\lambda)$ selection mechanism has the strongest selection pressure in several popular selection mechanism of genetic algorithm. VFMGA takes VF as a part of the mutation operator in RCGA. To individual that needs variation, respectively with uniform mutation operator and the VF algorithm mutate offspring to constitute a niche, and then apply (1+2) selection mechanism to merit as the final variation offspring from newly generated offspring and parent.

\section{Simulation Results}

We assume that 50 wireless sensor nodes are arranged in the square measuring area and its side length is $100 \mathrm{~m}$. In which the number of fixed sensor nodes is 30 , and the number of mobile sensor nodes is 20 . The measurement radius of all sensor nodes is $10 \mathrm{~m}$, and the communication radius of all sensor nodes is $20 \mathrm{~m}$. We use Matlab in a pc that its frequency is $3.0 \mathrm{GHZ}$ to simulate layout optimization of WSNs. Virtual force 


\section{Wireless Sensor Networks}

algorithm and genetic algorithm is sensitive at setting the parameter of algorithm, but there are no more authoritative guidelines for setting parameters. After a lot of experiments, we get the operating parameters of the network layout optimization algorithm as follows:

- VF algorithm: $w_{A}=4.01, w_{R}=1.56, d_{t h}=0.76 m, \quad$ MaxStep $=0.91 m, \quad F_{t h}=0.339$, and the number of iteration is 150 .

- $\quad$ RCGA algorithm: The population size is 100 , the crossover probability of heuristic crossover operator is set to 0.8 , the mutation probability of uniform mutation operator is set to 0.001 , and the algorithm runs 150 generations.

- VFGA algorithm:The following is operation parameters of the VF algorithm in first phase: $w_{A}=0.687$, $w_{R}=10, d_{t h}=0.5 m, \quad$ MaxStep $=0.55 m, \quad F_{t h}=0.165$, and the umber of iteration is 20. Operation parameters of RCGA algorithm are used to optimize the results in the second phase.

- VFCGA algorithm: $V=0.1$. One part of VF algorithm adopts the setting of (1), everytime crossover operation iterates one time, and other operating parameters are the same as the RCGA algorithm.

- VFMGA algorithm: Operating parameters of VF algorithm adopts the setting of (1), the process of each variation iterates one time, and other operating parameters are the same as the RCGA algorithm.

Fig. 1 shows the position of fixed sensor nodes after being initializated. Fig. 2, Fig. 3 and Fig. 4 respectively show the layout of sensor nodes after being optimized by VF algorithm, RCGA algorithm and VFGA algorithm. Fig. 5 shows the convergence of optimal placement algorithm. To further verify the performance of the algorithm, for each algorithm we make 20 times simulation optimization experiments for network layout. Table 1 shows the result of experiments. In which computation time and the number of iterations are the indicators of convergence. The average time-consuming of VFGA algorithm in first stage is $0.25 \mathrm{~s}$, and in Table 1 it is not added to the time-consuming of VFGA algorithm. In the process of layout optimization for wireless sensor network based on virtual force algorithm, the number of the fixed sensor nodes has a greater impact to the effects of network topology. When the size of network node is fixed, the network coverage rate will decreased with the proportion of the fixed nodes increased. At the same time, there is a harmonic vibration phenomenon similar to the pendulum at the vicinity of extreme points in the late time of optimization. The algorithm can not escape from local optimum and thus converge to the global optimum. The ratio of the number of the fixed sensor nodes from total number of nodes is $60 \%$, and the ratio of effective measurement area of the fixed sensor nodes from the size of the area under test is 58.52\%. It results that the average coverage rate of VF algorithm is only $83.79 \%$, and it is the lowest in these algorithms. From Fig. 5 we can observe that VF algorithm is difficult to converge in the late time of optimization process. Genetic algorithm has not the limitations at layout optimization. RCGA algorithm is designed in this paper reached $96.53 \%$ of the average coverage rate, and that is much higher than virtual force algorithm's. Because the inherent limitations of virtual force algorithm, so the result of VFCGA's layout optimization algorithm is weaker than RCGA's. The mutation operator based on virtual force in VFMGA algorithm enhances local search ability, but it can not effectively maintain the population's diversity because of the decrease of randomness. That leads VFMGA algorithm more easily prematurity than VFCGA algorithm. So the indicators of VFCGA algorithm are better than VFMGA algorithm in Table 1. In addition, either from the basic theory of genetic algorithms or the operation mode of evolution operator, the genetic algorithm has a strong dependence on the initial population. Only the initial population that has the characteristics of the solution space can make the algorithm fast approach the optimal solution by optimum way, and avoid premature convergence. Because of this, VFGA algorithm can generate higher-quality initial population by using virtual force in the first stage, and we can see from Table 1 that its average coverage rate reaches $83.79 \%$. The RCGA algorithm uses the strategy of randomly generating initial population at the case of a lot of fixed sensor nodes in monitoring area, and that result in the coverage area of Mobile sensor nodes and the fixed sensor nodes is overlap to some extent. Poor quality of the initial population reduces the efficiency of the follow-up the optimization process of the algorithm. Thus, VFGA algorithm still use exactly the same evolutionary strategy as RCGA algorithm in the second phase, but the average coverage rate is increased by $1 \%$ than the direct application of RCGA algorithm. 
We can know from Table 1 that VF algorithm's convergence rate is fastest, and the average computation time-consuming is 1.37s. Time-consuming of VFCGA algorithm and VFMGA algorithm are longer than RCGA algorithm and VFGA algorithm, because virtual force calculation is added in evolution operator. The average time-consuming of VFGA algorithm in the second phase is 27.53s longer than RCGA algorithm. If added time-consuming $0.25 \mathrm{~s}$ of VFGA algorithm in first stage, the average time-consuming of VFGA algorithm is 27.78s longer than RCGA algorithm. But the iterations number of VFGA algorithm is 18.5 times more than RCGA algorithm, and coverage rate increased by $1 \%$. So time loss is worth it. Therefore, overall speaking, Optimal speed and optimization efforts of VFGA algorithm achieve a good compromise.

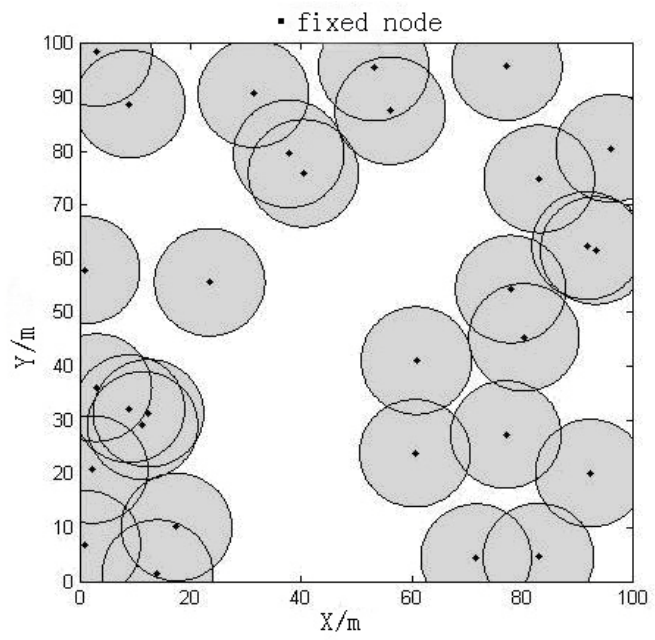

Figure1 Network layout initialized randomly

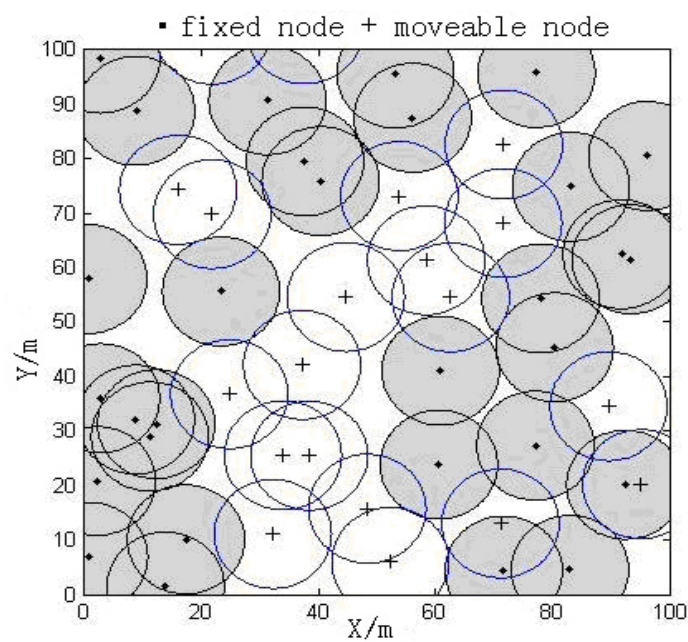

Figure 2. Network layout optimization of VF algorithm 
Wireless Sensor Networks

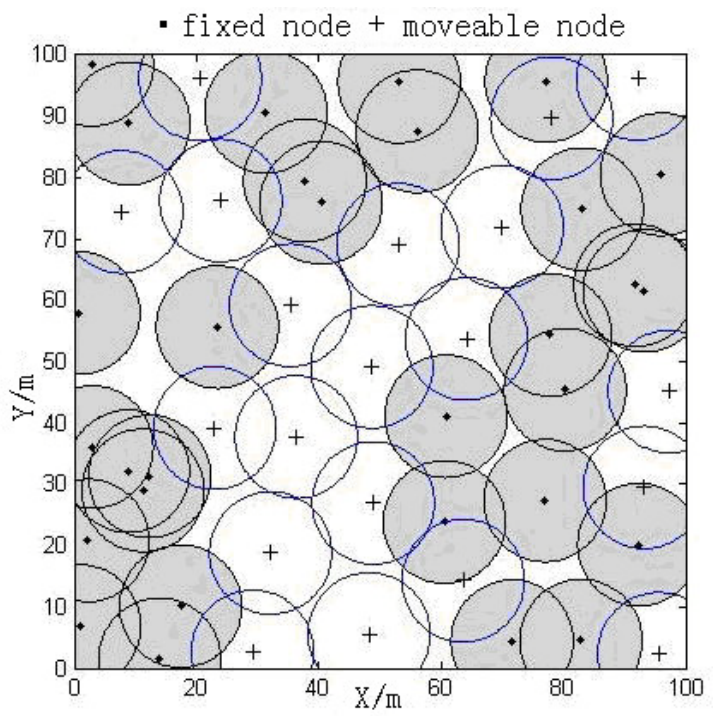

Figure 3. Network layout optimization of RCGA algorithm

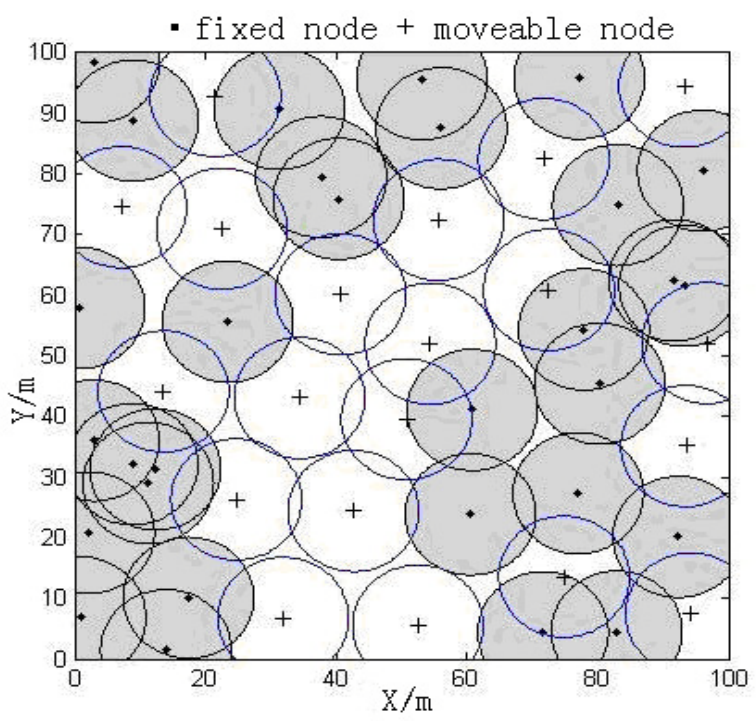

Figure 4. Network layout optimization of VFGA algorithm 


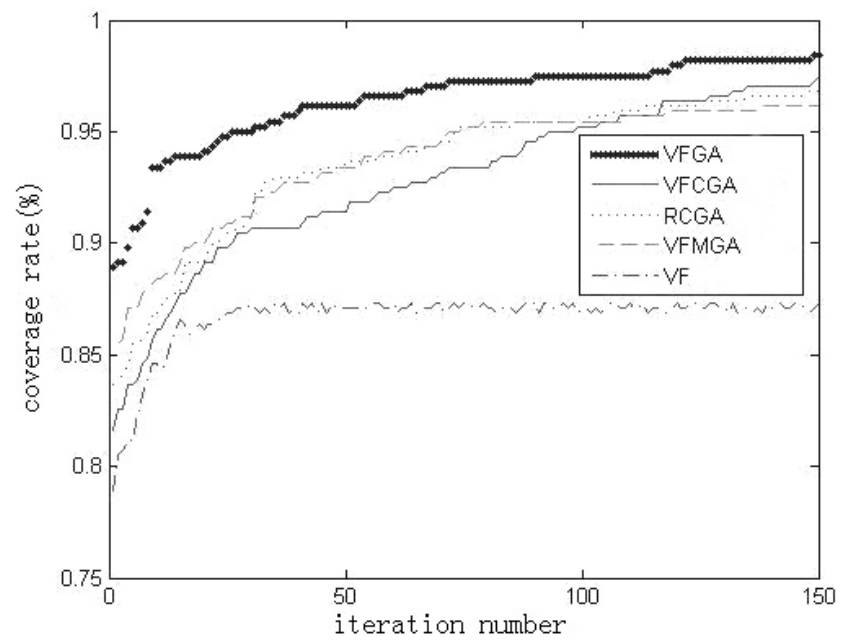

Figure 5. Comparison to the average performance of 20 times independent optimization

TABLE 1. COMPARISON TO THE AVERAGE PERFORMANCE OF 20 TIMES INDEPENDENT OPTIMIZATION

\begin{tabular}{|c|c|c|c|c|}
\hline \multicolumn{2}{|c|}{ Value } & $\begin{array}{c}\text { Coverage } \\
\text { Rate } \\
(\%)\end{array}$ & $\begin{array}{l}\text { Time- } \\
\text { Consuming } \\
\quad(s)\end{array}$ & $\begin{array}{c}\text { The } \\
\text { Number of } \\
\text { Iterations }\end{array}$ \\
\hline \multirow{3}{*}{ VF } & maximum & 87.30 & 1.66 & 30.0 \\
\hline & minimum & 80.95 & 1.28 & 8.0 \\
\hline & mean value & 83.79 & 1.37 & 15.4 \\
\hline \multirow{3}{*}{ RCGA } & maximum & 96.83 & 147.66 & 139.0 \\
\hline & minimum & 96.15 & 94.59 & 88.0 \\
\hline & mean value & 96.53 & 121.90 & 114.0 \\
\hline \multirow{3}{*}{ VFGA } & maximum & 98.41 & 172.02 & 149.0 \\
\hline & minimum & 96.83 & 114.72 & 104.0 \\
\hline & mean value & 97.53 & 149.43 & 132.5 \\
\hline \multirow{3}{*}{ VFCGA } & maximum & 97.51 & 230.09 & 150.0 \\
\hline & minimum & 94.33 & 147.45 & 100.0 \\
\hline & mean value & 95.83 & 198.48 & 133.8 \\
\hline \multirow{3}{*}{ VFMGA } & maximum & 96.15 & 251.17 & 150.0 \\
\hline & minimum & 93.89 & 155.20 & 92.0 \\
\hline & mean value & 95.17 & 215.87 & 129.9 \\
\hline
\end{tabular}


Wireless Sensor Networks

\section{Conclusions}

For the the problems of virtual force algorithm and genetic algorithm for coverage optimization in wireless sensor networks, this paper proposes a two-stage dynamic sensor deployment strategy in WSNs based on virtual force and genetic algorithm. That is, the algorithm firstly deploys the dynamic sensors in continuous larger area in WSNs in an approximate optimal way to produce high quality initial population by virtual force. Then GA is employed to achieve global optimization coverage of WSNs based on the result of the first stage. Simulation results demonstrate that the algorithm presented in this paper is effective and efficient. Our next research includes two respects: one is to reduce the difficulty of parameter setting, and the other is to research a more effective combination way of virtual force algorithm and genetic algorithm.

\section{Acknowledgements}

This work was supported by the Science and Technology Foundation of Zhongshan (Grant no. 409S29) and the Science and Technology Foundation of University of Electronic Science and Technology of China, Zhongshan Institute (Grant no. 2007YKQ18).

\section{References}

[1] Wang G L, Cao G H, Porta T L. "Movement-assisted sensor deployment," Proceedings of the 23rd Conference of the IEEE Computer and Communications Societies, Hong Kong, 2004, pp.2469-2479.

[2] Howard A, Mataric M J, Sukhatme G S., "Mobile sensor network deployment using potential fields:A distributed, scalable solution to the area coverage problem," Proceedings of the 6th International Symposium on Distributed Autonomous Robotics Systems, Fukuoka, Japan, 2002, pp. 299-308.

[3] Zou Y, Chakrabarty K., "Sensor deployment and target localization in distributed sensor networks," ACM Transactions on Embedded Computing Systems, vol. 3, no. 1, 2004, pp. 61-91.

[4] Tian Yiming, Lu Yang, Wei Zhen, Wu Qilin, "Research on energy-efficient optimization for coverage control in wireless sensor networks,” Journal of Electronic Measurement and Instrument, vol. 23, no. 11, 2009, pp. 65-71.

[5] WANG Xue, WANG Sheng, MA Jun-jie, "Dynamic Sensor Deployment Strategy Based on Virtual ForceDirected Particle Swarm Optimization in Wireless Sensor Networks,” Acta Electronica Sinica, vol. 35, no. 11, 2007, pp. 2038-2042(in Chinese).

[6] JIA Jie, CHEN Jian, CHANG Gui-ran, etc., "Optimal coverage scheme based on genetic algorithm in wireless sensor networks,” Control and Decision, vol. 22, no. 11, 2007, pp. 1289-1292(in Chinese).

[7] LI Hong, JIAO Yong-chang, ZHANG Li, WANG Yu-ping, "Novel hybrid genetic algorithm for global optimization problems,” Control Theory \& Applications, vol. 24, no. 3, 2007, pp. 343-348(in Chinese).

[8] Tan G, Jarvis S A, Kermarrec A M., "Connectivity-guaranteed and obstacle-adaptive deployment schemes for mobile sensor networks,” IEEE Transactions on Mobile Computing, vol. 8, no. 6, 2009, pp. 836-848.

[9] Zhou Pucheng, Cui Xunxue, Wang Shumin etc., "Virtual Force-based Wireless Sensor Network Coverageenhancing Algorithm,” Journal of System Simulation, vol. 21, no. 5, 2009, pp. 1416-1419.

[10] YANG Ming-hua, CAO Yuan-da , TAN Li etc., “A New Mechanism of Deployment and Management in Mobile Sensor Network,” Journal of Beijing Institute of Technology, vol. 28, no. 12, 2008, pp. 1074-1077 